\title{
Vogt-Koyanagi-Harada syndrome associated to hypothyroidism revealed by myocarditis
}

\author{
F. Ben Fredj Ismail, A. Mzabi, M. Karmani, A. Rezgui, H. Mhiri, C. Laouani-Kechrid
}

Department of Internal Medicine, Sahloul Hospital, Sousse, Tunisia Email: bfi.fatma@yahoo.fr

Received 6 April 2012; revised 4 May 2012; accepted 23 July 2012

\begin{abstract}
Purpose: Vogt-Koyanagi-Harada (VKH) syndrome is usually defined as an uveo-meningitis who may be associated with auditory and cutaneous signs. Association of VKH syndrome and autoimmune thyroiditis is uncommon. Observation: A 28-year-old man was admitted with thoracic pain due to myocarditis. Two years ago he presented VKH syndrome with specific ocular manifestation and deafness, treated by corticosteroids and immunosuppressive. Etiologic investigation of myocarditis concluded to deep hypothyroidism related to Hashimoto thyroiditis. The patient improved after substitutive treatment by thyroid hormones. Conclusion: Thyroid function should be systematically investigated in case of VKH syndrome and particularly when associated to dysthyroidism symptoms.
\end{abstract}

Keywords: Vogt-Koyanagi-Harada; Myocarditis; Hypothyroïdism; Hashimoto Thyroïditis.

\section{INTRODUCTION}

Vogt-Koyanagi-Harada (VKH) Syndrome is usually defined as an uveo-meningitis who may be associated with auditory and cutaneous signs. It primarily affects Asiatic people but also African and Caucasian ones. It would be associated with certain HLA class II antigens. Its pathogenesis is probably autoimmune; however, its association with other autoimmune diseases such as Hashimoto's thyroiditis is rare.

\section{AIM}

We report a new case with this original rare association on the one hand and the revelation of thyroiditis by myocarditis on the other hand.

\section{OBSERVATION}

A North African man of 28 years old was admitted to emergency exploration for acute chest pain preceded by a physical asthenia lasting for one month. Two years earlier he had presented a VKH syndrome whose diagnosis was retained because the presence of a bilateral granulomatous uveitis with retinal detachment, retinal ischemia and areas of sensorineural hearing loss. The patient received high-dose steroid therapy and six courses of cyclophosphamide monthly and had unexpectedly stopped his treatment. Physical examination on admission was a discrete motor weakness of the pelvic girdle and reduced tendon reflexes. Cardiopulmonary auscultation was normal. The electrocardiogram showed signs of subepicardial ischemia in the apical-lateral territory. The biological noted an increase in creatine phosphokinase (CPK) to $2290 \mathrm{IU} / \mathrm{l}$ and lactate dehydrogenase (LDH) to $910 \mathrm{IU} /$; troponin, triglycerides, total cholesterol, blood counts and parameters inflammation were within the limits of normal. Echocardiography noted diffuse hypokinesis of the left ventricle without impaired ejection fraction or other cardiac abnormalities. Myocardial scintigraphy showed a diffuse hypo perfusion stress. As part of this etiological and myocardial muscle, a thyroid showed peripheral hypothyroidism (TSH $>100$ $\mathrm{mIU} / \mathrm{l}$ and T4 $0.1 \mathrm{ng} / \mathrm{dl})$. The anti-peroxidases were present at high titer. No signs of adrenal insufficiency were noted and the basic cortisol was normal. The patient was then treated with substitutive thyroid hormone plus captopril established for myocarditis. Its evolution was favorable with clinical improvement and normalization of laboratory tests. Control echocardiography was normal. Ophthalmologic examination had found control of VKH disease in remission, not requiring the resumption of steroid therapy.

\section{DISCUSSION}

VKH syndrome usually affects young adults between 30 and 40 years with a female predominance $[1,2]$. Its clinical manifestations is various, they evolve classically into three phases. A phase of meningitis can occur with headache, meningeal irritation, seizures, neurological deficit, hearing loss and psychiatric signs. Second phase is characterized by ophthalmic pain and decreased visual 
acuity with anterior uveitis, granulomatous or not, associated with serous retinal detachment and papillitis. Involvement is bilateral in almost all cases and can be asymmetrical. This phase can last from several months to several years. The most common complications are cataract and glaucoma with risk of blindness [1]. The convalescent phase is marked by a diffuse depigmentation of the fundus and the appearance of skin symptoms in type of alopecia, poliosis (hair's discoloration) and vitiligo.

The pathogenesis of this syndrome is still little known; an autoimmune mechanism is very likely as suggested by the presence of $\mathrm{T}$ CD4+ cells reactive to certain proteins of melanocytes in the blood, cerebrospinal fluid, the choroid and the skin of patients with active disease [3]. A particular genetic predisposition, HLA-DRB1 0405, appears to predispose to this condition.

The association of VKH syndrome with an autoimmune disease of organs, especially of the thyroid is rare, isolated cases have been reported in the literature. This was mainly Hashimoto's disease [4-8], more rarely with Graves' disease [9] or polyglandular syndrome [10]. The pathogenesis of Hashimoto's thyroiditis appears to involve humoral immunity as evidenced by the presence of anti-thyroid, but also cellular immunity. Indeed, they are clones T CD4+ cells specific for certain antigens (thyroglobulin and microsomal antigen major or TPO) that appear to play an important role in the destruction of thyroid epithelial cells. Abnormalities of immune regulation have also been observed with a decrease in circulating T CD8+ cells with suppressive function [11]. A genetic susceptibility characterized by different alleles, including HLA-DR3 in particular, has been associated with Hashimoto's disease. Pathophysiological similarities exist therefore between these two autoimmune diseases; their association in the same patient would not be a coincidence. The second peculiarity of the case reported in this work is the mode of presentation of hypothyroidism. Indeed, the non-ischemic myocardial injury is rare in hypothyroidism which accompanies most of pericarditis [12]. This achievement, in type of hypo kinesis septal or dilated cardiomyopathy, is caused by reversible myocardial functional impairment. In Hashimoto's thyroiditis, lymphocytic myocarditis of probable autoimmune origin was reported in an autopsy study [13]. Treatment of VKH syndrome is based on steroids and immunosuppressors and treatment of hypothyroidism is substitutive thyroid hormone. Captopril establishes myocarditis; when due to hypothyroidism, it is also reversible when hormonal disorder is early corrected $[1,7,11,13]$.

\section{CONCLUSION}

$\mathrm{VKH}$ syndrome is a systemic disease rare, probably autoimmune origin can be associated with other autoimmune diseases of organs such as Hashimoto's thyroiditis. This is to systematically seek to establish an early diagnosis and appropriate treatment.

\section{REFERENCES}

[1] J.-M. Guenoun, C. Parc, R. Dhote and A. P. Brezin, "Le Syndrome DE Vogt-Koyanagi-Harada: Aspects Cliniques, Traitement et Suivi à Long Terme dans UNE Population caucasienne et Africaine,"Journal Francais d'ophtalmologie, Vol. 27, No. 9, 2004, pp.1013-1016. doi:10.1016/S0181-5512(04)96257-2

[2] F.-Z. Alaoui, S. Benamour, H. El Kabli and A. Amraoui, "Syndrome de Vogt-Koyanagi-Harada. A propos de huit cas,” La Revue de Médecine Interne, Vol. 28, No. 4, 2007, pp. 250-254. doi:10.1016/j.revmed.2006.12.009

[3] F. M. Damico, F. T. Bezerra, G. C. da Silva, F. Gasparin and J. H. Yamamoto, "New Insights into Vogt-KoyanagiHarada Disease,” Arquivos Brasileiros de Oftalmologia, Vol. 72, No. 3, 2009, pp. 413-420. doi:10.1590/S0004-27492009000300028

[4] P. Wiesli, W. Bernauer and J. Furrer, "Headache and Bilateral Visual Loss in a Young Hypothyroid Indian Man,” Journal of Endocrinological Investigation, Vol. 22, No. 2, 1999, pp. 141-143.

[5] N. Jaggarao, D. Voth and J. Jacobsen, "The Vogt-Koyanagi-Harada Syndrome: Association with Hypothyroiddism and Diabetes Mellitus," Postgraduate Medical Journal, Vol. 65, No. 766, 1989, pp. 587-588. doi:10.1136/pgmj.65.766.587

[6] N. Kluger, F. Mura, B. Guillot and D. Bessis, "VogtKoyanagi-Harada Syndrome Associated with Psoriasis and Autoimmune Thyroid Disease," Acta Dermato-Venereologica, Vol. 88, No. 4, 2008, pp. 397-398.

[7] H. I. Chi, M. Furue and Y. Ishibashi, "Vogt-KoyanagiHarada's Syndrome Associated with Hashimoto's Thyroiditis," The Journal of Dermatology, Vol. 21, No. 9, 1994, pp. 683-686.

[8] M. P. Paroli, M. Pinca, S. Speranza, M. Marino and P. Pivetti-Pezzi, "Paracentral corneal melting in a patient with Vogt-Koyanagi-Harada’s syndrome, psoriasis, and Hashimo-to's thyroiditis," Ocular Immunology and Inflammation, Vol. 11, 2003, pp. 309-313. doi:10.1076/ocii.11.4.309.18268

[9] J. H. Seo, H. G. Yu and H. Chung, "A Case of Vogt-Koyanagi-Harada Disease in a Patient with Graves Disease," Korean Journal of Ophthalmology, Vol. 23, No. 2, 2009, pp. 112-113. doi:10.3341/kjo.2009.23.2.112

[10] N. S. Jovic, M. Nesovic, D. N Vranjesevic, J. Ciric, D. M. Marinkovic and B. Bonaci, "The Vogt-Koyanagi-Harada Syndrome: Association with Autoimmune Polyglandular Syndrome Type 1,” Postgraduate Medical Journal, Vol. 72, No. 850, 1996, pp. 495-497. doi:10.1136/pgmj.72.850.495

[11] F. Duron, E. Dubosclard and C. Johanet, "Thyroïdites," EMC-Endocrinologie, Vol. 1, No. 1, 2004, pp. 3-18.

[12] L. Guillevin, C. Scheuble, J.-R. Attali, E. Modigliani and J. Sebaoun, "Etude Echocardiographique des Hypothyroïdies,” La Revue de Médecine Interne, Vol. 2, No. 2, 
1981, pp. 187-194. doi:10.1016/S0248-8663(81)80065-3

[13] G. Grandmaison, M. Izembart, P. Fornes and F. Paraire,

"Myocarditis Associated with Hashimoto's Disease: A
Case Report,” International Journal of Legal Medicine, Vol. 117, No. 6, 2003, pp. 361-364.

doi:10.1007/s00414-003-0392-5 